\title{
MULTI-FREQUENCY VLBI OBSERVATIONS OF B0218+357
}

\author{
RICHARD W. PORCAS AND ALOK R. PATNAIK \\ Max-Planck-Institut für Radioastronomie \\ Auf dem Hügel 69, D-53121 Bonn, Germany.
}

\begin{abstract}
We present the results from VLBI observations at three frequencies of the gravitational lens system B0218+357. From the source double structure, seen in both the $\mathrm{A}$ and $\mathrm{B}$ images at $15 \mathrm{GHz}$, we have derived a relative magnification matrix, and we show that the lens mass distribution must be non-spherical. We investigate how far the matrix parameters derived from $15 \mathrm{GHz}$ observations can be used to relate the $\mathrm{A}$ and $\mathrm{B}$ images at 1.7 and $5 \mathrm{GHz}$, where the image sizes are much larger.
\end{abstract}

\section{Introduction}

The gravitational lens system B0218+357 (Patnaik et al. 1993) consists of two flat-spectrum radio image components separated by 335 mas, and an Einstein ring of similar diameter whose centre is slightly offset from the B (fainter) image. Since its discovery as a lensed system it has been subject to intensive study at both optical and radio wavebands, especially with the hope that the lens mass - of single galaxy size - may have a simple distribution. Measurement of this, taken together with a time delay between image variations, could lead to a direct determination of the Hubble constant (Refsdal, 1964). The redshift of the lens is well established as 0.6847 from optical and radio absorption measurements, and a preliminary estimate of the background object redshift is 0.96 (e.g. Lawrence in these proceedings). Corbett et al. (these proceedings) has reported a value of ca. 12 days for the time delay, from VLA measurements of variations in the image percentage polarization.

\section{VLBI observations at $15 \mathrm{GHz}$}

Determination of the image relative magnification matrix by VLBI studies of the mas structure of the images can help constrain the lens mass dis- 

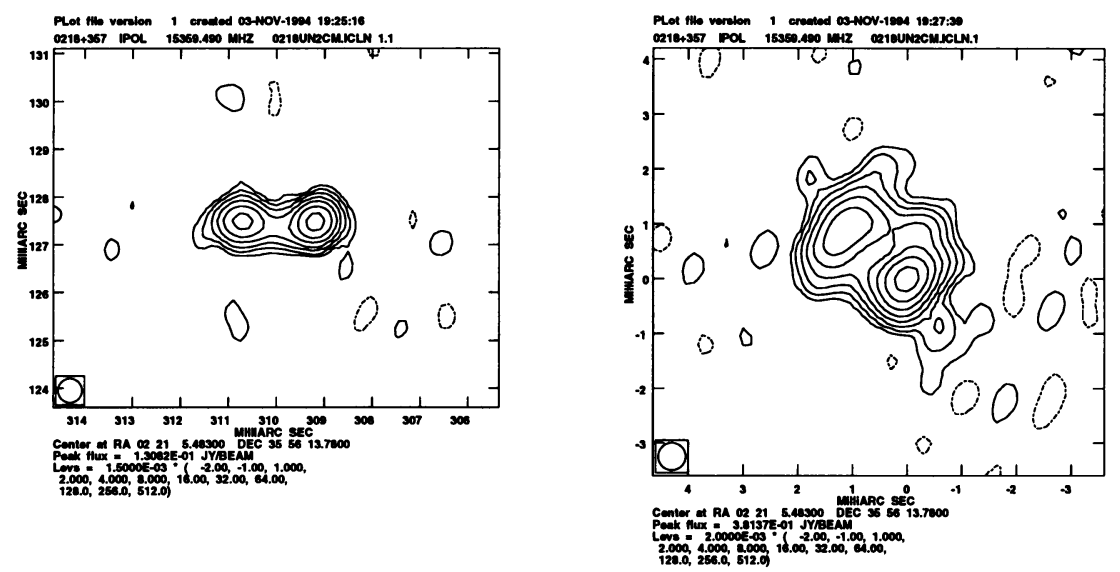

Figure 1. First epoch $15 \mathrm{GHz}$ map of $\mathrm{B} 0218+357 \mathrm{~B}$ (left) and A (right), resolution 0.5 mas.

tribution, and a number of VLBI observations at 1.7 and $5 \mathrm{GHz}$ have been published (Patnaik et al. 1993, 1994, Shaffer 1994, Polatidis et al. 1995, Xu et al. 1995), At these frequencies, however, it seems impossible to disentangle the contributions from intrinsic source structure and lens distortion in the observed image morphologies. We made observations with the VLBA in 1994 October at $15 \mathrm{GHz}$, a frequency a factor 3 higher, and a resolution a factor 2 higher, than any previous VLBI observations. Details of the observations are described in Patnaik et al. (1995), and maps of the A and $\mathrm{B}$ images with $0.5 \mathrm{mas}$ resolution are shown in Fig. 1. The intrinsic double structure of the source at $15 \mathrm{GHz}$ is clearly seen in both images, the component flux ratio being 1.64 in both $\mathrm{A}$ and $\mathrm{B}$. Some striking features of these VLBA maps are the difference in the component-separation position angles in the two images, and also the elongation of both components in $\mathrm{PA}-40^{\circ}$ in the $\mathrm{A}$ image. This extension of image $\mathrm{A}$ is also seen in maps at 1.7 and $5 \mathrm{GHz}$, and corresponds to the expected 'tangential stretching' expected for the exterior image in this system (Narasimha and Patnaik 1994).

These observations of $\mathrm{B} 0218+357$ seem to provide a 'textbook' case which exemplifies gravitational lens properties. The equality of the component ratio in the two images shows that the magnification is uniform over the region of the $15 \mathrm{GHz}$ structure. Gaussian fits to the more resolved component yield deconvolved sizes whose ratio between the images $(\mathrm{A} / \mathrm{B}=3.9 \pm 0.6)$ agrees well with the flux ratio $(\mathrm{A} / \mathrm{B}=3.64)$, showing that the surface brightness of this component is the same in A and B. This system also demonstrates the interesting property that, although the action of the lens produces a change of the component-separation position angle between the images, the position angle of linear polarization (corrected for 

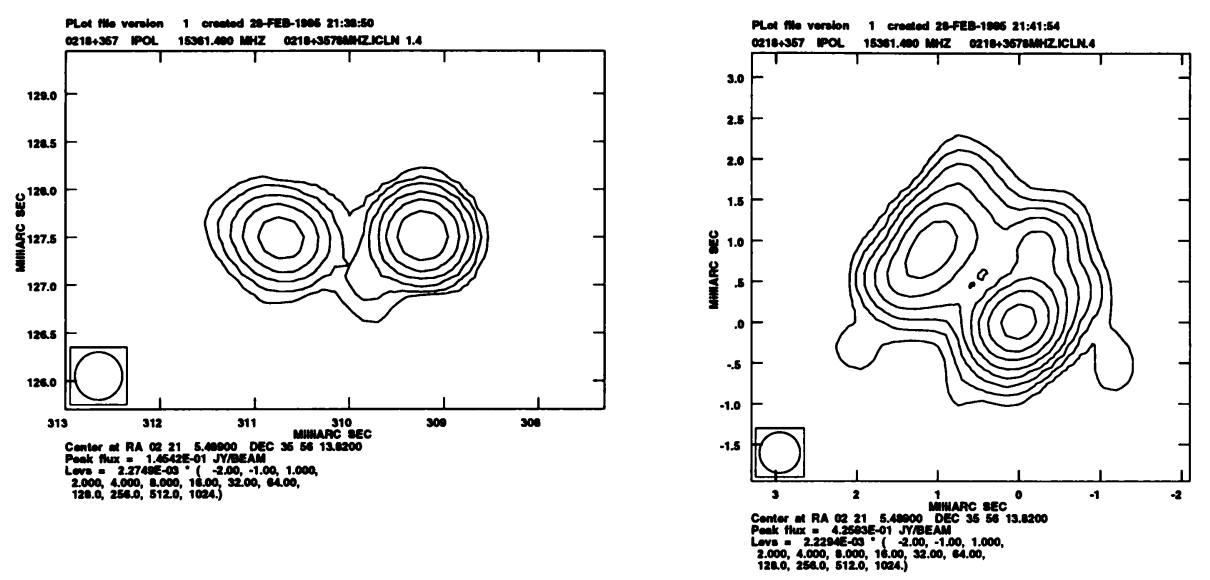

Figure 2. Second epoch $15 \mathrm{GHz}$ map of $\mathrm{B} 0218+357 \mathrm{~B}$ (left) and $\mathrm{A}$ (right), resolution 0.5 mas.

Faraday rotation) is the same in both images. Finally, we have been able to determine a relative magnification matrix, describing the mapping of the $\mathrm{B}$ image into $\mathrm{A}$, using the image flux ratio and the transformation of the component separation vector (Patnaik et al. 1995). The properties of this matrix show that the lens mass distribution is non-spherical.

Further observations of B0218+357 were made with the VLBA at $15 \mathrm{GHz}$ in 1995 January, as part of the survey of Patnaik et al. (these proceedings). The maps of A and B are presented in Fig 2; they have a resolution of 0.5 mas. They show morphologies very similar to those from the 1994 October observations, and, in particular, there is no significant change in the component separations in either image. However, the faint extension of the 'core' (western) component at the lowest contour levels in PA ca. $-10^{\circ}$ seen in 1994 October has become a much more prominent, well-defined feature in 1995 January, presumably indicating the ejection of a new 'jet' component. Traces of this new component can also now be seen in the B image, as an extension of the lowest contours in PA ca. $135^{\circ}$. (on the opposite side of the main component separation line, due to opposite parity of the images). We have used this exciting new development to furnish a new constraint to refine our previous values for the elements of the image relative magnification matrix.

\section{VLBI observations at 1.7 and $5.0 \mathrm{GHz}$}

We have also made global VLBI (MK3) observations of B0218+357 at 1.7 and $5.0 \mathrm{GHz}$, in 1992 June and March. Details of the observations will be published elsewhere. The $5.0 \mathrm{GHz}$ maps of images $\mathrm{A}$ and $\mathrm{B}$ (resolution 1 

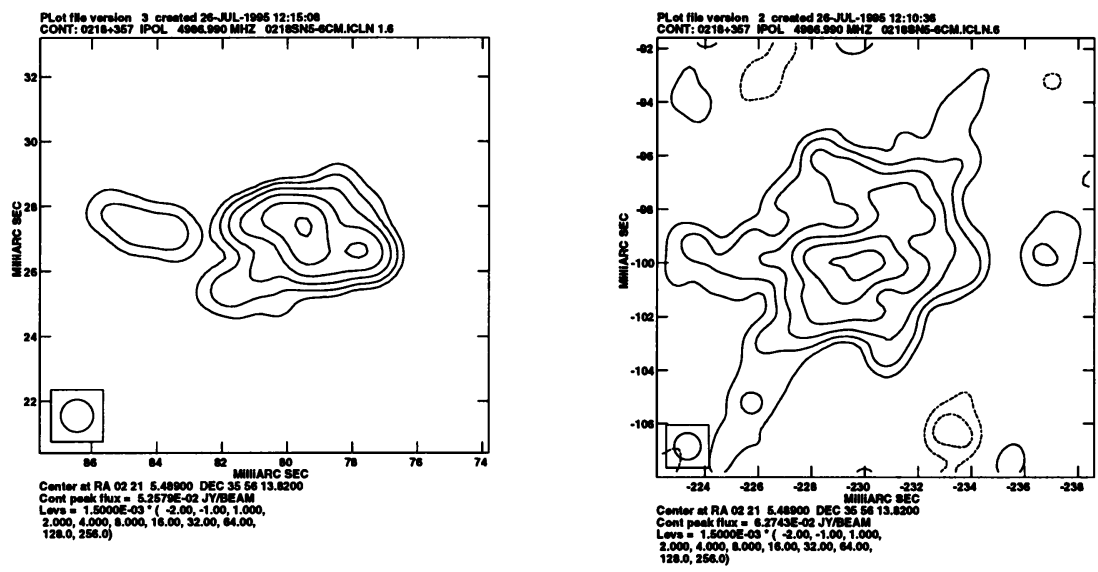

Figure 3. $5 \mathrm{GHz}$ maps of $\mathrm{B} 0218+357 \mathrm{~B}$ (left) and $\mathrm{A}$ (right) with 1mas resolution.

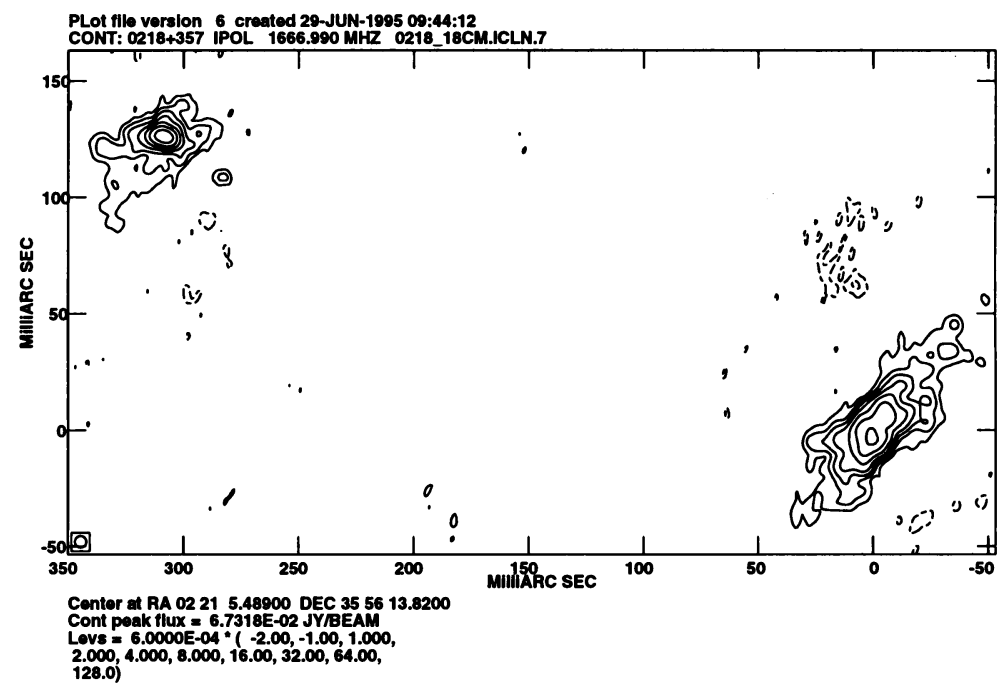

Figure 4. $1.7 \mathrm{GHz}$ map of $\mathrm{B} 0218+357$ with 5 mas resolution

mas) are presented in Fig. 3 and the $1.7 \mathrm{GHz}$ map of both images (resolution $5 \mathrm{mas}$ ) is presented in Fig.4. At these frequencies the angular resolution is not sufficient to easily identify the 1.4 mas double structure seen at $15 \mathrm{GHz}$, but both observations show a 'radial' extension of image B, and the tangential extension of image $\mathrm{A}$ in $\mathrm{PA}-40^{\circ}$, so prominent at $15 \mathrm{GHz}$. The image sizes also increase with increasing wavelength. 
Although the registration of VLBI maps made at different frequencies usually presents difficulties, knowledge of the relative magnification matrix, and measurement of the precise relative separation between corresponding features in two images, can lead to a unique registration (Porcas and Patnaik 1995). Using this method we find that the peaks of the $5 \mathrm{GHz}$ image maps lie close to a point between the two components seen at $15 \mathrm{GHz}$. At $1.7 \mathrm{GHz}$, the separation of the centroid of the brightness distributions of the images (which are corresponding points) locates them very close to the eastern, more resolved component at $15 \mathrm{GHz}$.

The method used above assumes that the same relative magnification matrix can be used for these maps at all 3 frequencies; this assumption may break down when the image sizes become large compared with the scale size of the lens potential. As a crude test, we measured the size of the $5 \mathrm{GHz}$ images (at the $3 \mathrm{rd}$ contour down from the peak) in the two directions corresponding to the $15 \mathrm{GHz}$ matrix eigenvector directions. The ratio of the $A$ and $B$ image sizes in these directions do indeed agree very well with the ratios from the matrix. This confirms that the achromatic property of gravitational lensing, and small source size, result in the same matrix being applicable to our $5 \mathrm{GHz}$ maps. At $1.7 \mathrm{GHz}$ the image sizes are very much larger. We have measured the ratio between the areas of image $\mathrm{A}$ and $B$ at two different contour levels. At the $2.3 \mathrm{mJy} /$ beam level, the ratio (2.65) agrees well with the flux density ratio (2.61) between $\mathrm{A}$ and $\mathrm{B}$ at $1.7 \mathrm{GHz}$, showing the same surface brightness in the images at the largest angular scales. This ratio is known to be frequency dependent, however, and presumably demonstrates the breakdown of the assumption of small image size at $1.7 \mathrm{GHz}$. For surface brightness $>5 \mathrm{mJy} /$ beam, the ratio of areas (3.56) differs significantly from the flux ratio (2.77), however, and the peak brightness in image B is higher than in image A. Although this can arise, in principle, if there is compact structure in both A and B which is unresolved in different directions, it is unclear whether this explanation applies here. Nair (these proceedings) has suggested an alternative explanation for this effect.

Acknowledgements We thank Drs. Ian Browne and Sunita Nair for lively conversations regarding the image structures of this intriguing lens system.

\section{References}

Narasimha, D. \& Patnaik, A.R., 1994, in Gravitational lenses in the Universe, eds. Surdej et al., (Liège: Université de Liège), 295

Patnaik, A.R., Browne, I.W.A., King, L.J., Muxlow, T.W.B., Walsh, D. \& Wilkinson, P.N., 1993, MNRAS, 261, 435

Patnaik, A.R., Porcas, R.W., Browne, I.W.A., Muxlow, T.W.B., Narasimha, D., 1994, in Compact Extragalactic Sources, eds. Zensus \& Kellermann, NRAO, 129 
Patnaik, A.R., Porcas, R.W. \& Browne, I.W.A, 1995, MNRAS, 274, 5p

Polatidis, A.G., Wilkinson, P.N., Xu, W., Readhead, A.C.S., Pearson, T.J., Taylor, G.B. \& Vermeulen, R.C., 1995, ApJSup, 98, 1

Porcas, R.W. \& Patnaik, A.R., 1995, in 10th working Meeting on European VLBI for Geodesy and Astrometry, Matera, in press

Refsdal, S., 1964, MNRAS, 128, 307

Shaffer, D.B. 1994, in Compact Extragalactic Sources, eds. Zensus \& Kellermann, NRAO, 132

Xu, W., Readhead, A.C.S., Pearson, T.J., Polatidis, A.G., \& Wilkinson, P.N., 1995, ApJSup, in press 